\title{
Research Paper: The Correlation Between Rates of Falling, Balance, Quality of Life and Fear of Falling in Patients With Chronic Stroke
}

\author{
Hamid Azadeh ${ }^{1} \odot$, Atefeh Fekri², Hamid Amraie ${ }^{3}$, Meysam Roostaei ${ }^{4},{ }^{*}$ Hamzeh Baharlouei $^{5}$
}

1. Musculoskeletal Research Center, Isfahan University of Medical Sciences, Isfahan, Iran.

2. Student Research Committee, Tabriz University of Medical Sciences, Tabriz, Iran.

3. Department of Physiotherapy, School of Rehabilitation Sciences, Isfahan University of Medical Sciences, Isfahan, Iran.

4. Department of Occupational Therapy, School of Rehabilitation, Tehran University of Medical Sciences, Tehran, Iran.

5. Research Center for Musculoskeletal Rehabilitation, Ahvaz Jundishapur University of Medical Sciences, Ahvaz, Iran.

Received: 07 Aug. 2017 Accepted: 26 Nov. 2017

Keywords:

Stroke, Quality of

life, Fear of falling, Balance
Cltation: Azadeh H, Fekri A, Amraie H, Roostaie M, Baharlouei H. [The Correlation Between Rates of Falling, Balance, Quality of Life and Fear of Falling in Patients With Chronic Stroke (Persian)]. Archives of Rehabilitation. 2018; 19(1):36-43. https:// doi.org/10.21859/JREHAB.19.1.36

\section{https://doi.org/10.21859/JREHAB.19.1.36}

\section{A BSTRACT}

Objective Stroke is one of the most important causes of disability worldwide. Fear of falling is one of the consequences of stroke that could decrease the quality of life. It presents in the patients who have fallen as well as in patients had not experienced a fall. Fear of falling is essential because of its impact on physical and mental health and even the quality of life. In addition to adverse effects of fear of falling on the patient, it may have a significant impact on the family quality of life. There are few published studies about the fear of falling after stroke, but given its significant implications for quality of life in patients, more studies are needed to improve the knowledge about the correlation of fear of falling and quality of life. The findings could help identify people at risk and also develop the prevention strategy in the management of fear of falling and quality of life. Previous studies assessed the acute and subacute patients with stroke and the authors could not find any research conducted with chronic subjects. Also, they measured the correlation between fear of falling and personal characteristics of participants and there is lack of evidence about the correlation between fear of falling with the rate of falling, postural control and SF-36. The objective of the current study was to examine the relationships between the fear of falling and rate of falling, balance, and quality of life in patients with chronic stroke.

Materials \& Methods The subjects ( $n=44,20$ males and 24 females) were selected based on the convenience sampling method from the patients who referred rehabilitation clinics. The inclusion criteria were individuals within at least six months after the stroke, unilateral signs, ability to walk independently and the exclusion criteria were metabolic or rheumatologic disease, lower extremity deformities, cognitive impairments and visual impairments. The participants completed a demographic form consisting of age, gender, height, weight, comorbidities, education, and the duration of the stroke, affected side, dominant side and rate of falls in last year. The fear of the falling and quality of life was measured using Fall Efficacy Scale- International and SF-36, respectively. Functional reach test and timed up and go were conducted for balance assessing. The Spearman's correlation was used to find whether there are significant relationships between fear of falling and other variables.

Results The Pearson analysis showed correlation between Fall Efficacy Scale- International and timed up and go $(P<0.001)$ and Functional reach test $(P=0.03)$ scores and some subscales of $S F-36$ including physical functioning $(P<0.001)$, role limitations due to physical health $(P<0.001)$ and physical component $(P<0.001)$. However, this association was not observed with the rate of falling $(P=0.015)$, pain $(P=0.42)$, general health $(P=0.12)$, energy $(P=0.09)$, social function $(P=0.30)$ and role limitations due to emotional problems $(P=0.24)$ and metal component $(\mathrm{P}=0.41)$.

Conclusion The findings of the present study demonstrated the correlation between balance, physical dimensions of quality of life and fear of falling in patients with stroke; however, the rate of falling has no association with fear of falling.

\section{* Corresponding Author:}

Address: Research Center for Musculoskeletal Rehabilitation, Ahvaz Jundishapur University of Medical Sciences, Ahvaz, Iran.

Tel: +98 (61) 33743505

E-Mail: baharlooh@gmail.com 


\title{
بررسى ارتباط ترس از زمينخوردن با تعداد دفعات زمين خوردن، تعادل و كيفيت زندكّى در بيماران

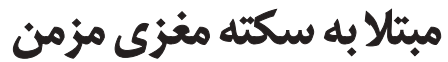

\author{
حميد آزاده' فه، عاطفه فكرى"، حميد امرائى"، ميثم روستايى" "حمزه بهارلويى"

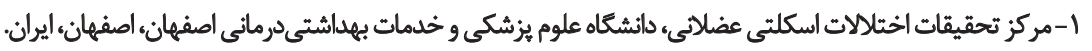

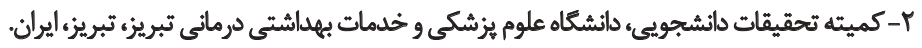

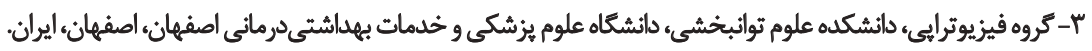

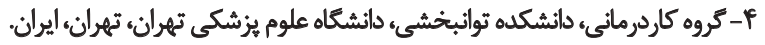

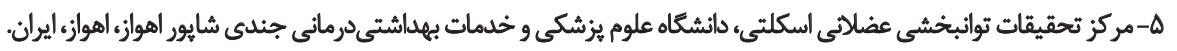

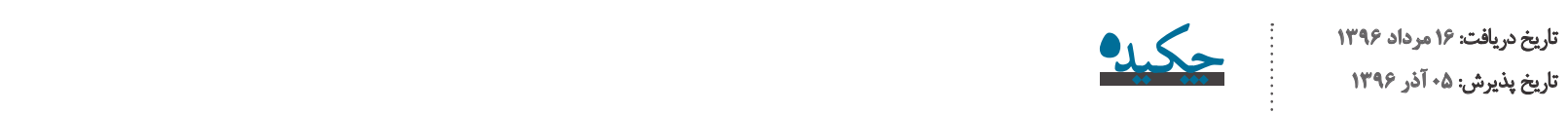

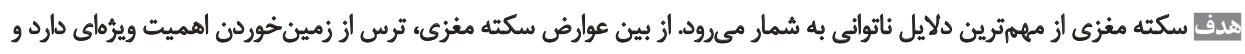

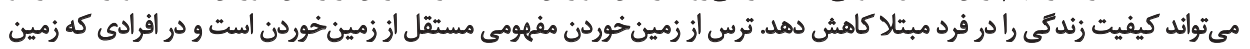

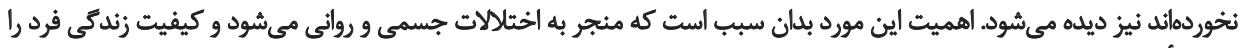

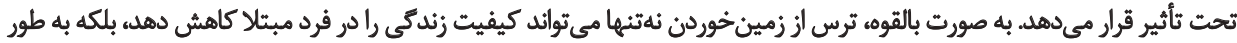

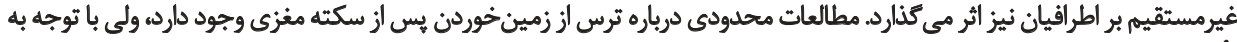

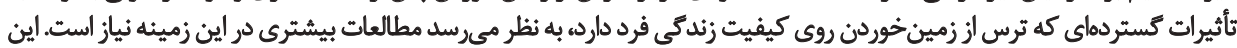

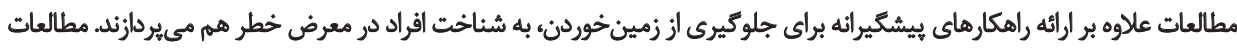

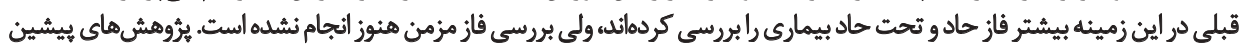

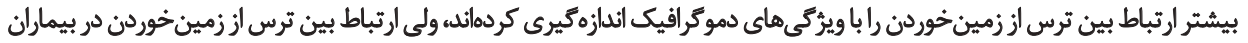

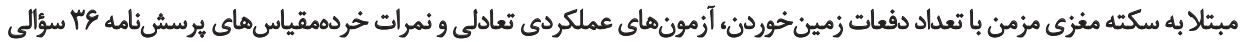

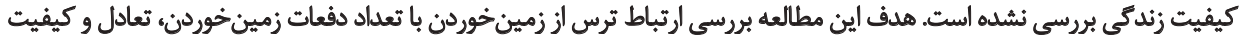

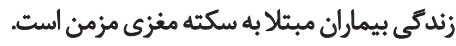

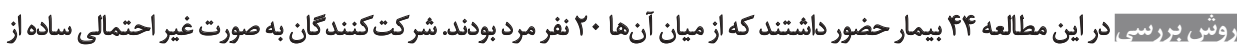

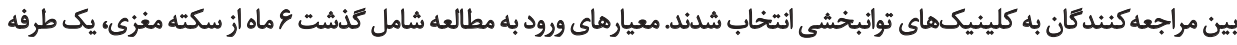

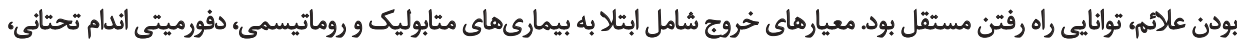

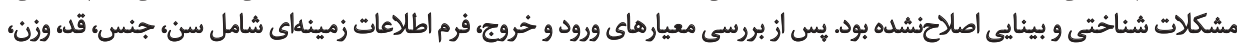

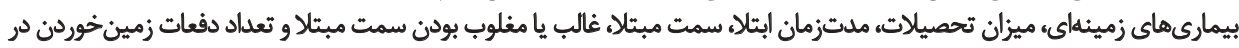

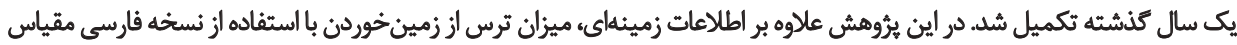

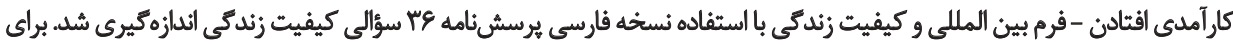

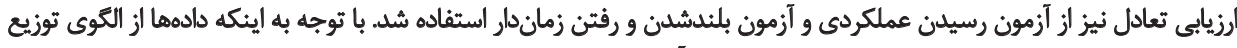

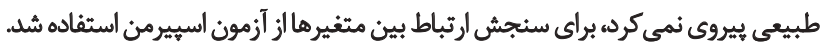

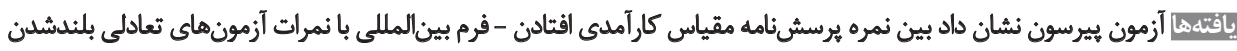

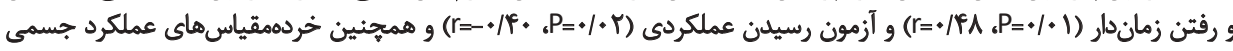

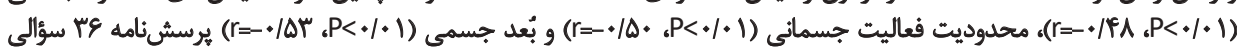

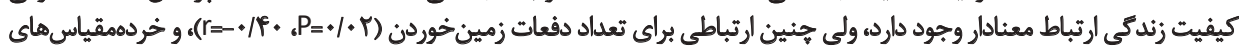

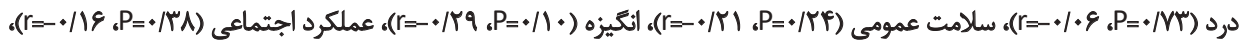

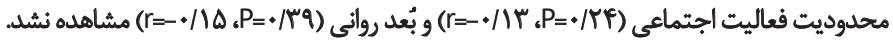

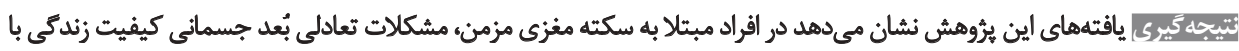

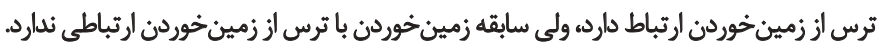

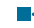

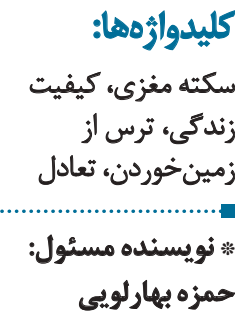

نشائى: اهوازء، دانشكاه علوم يزُشكى وخدمات بهداشتى درمانى جندى شايور اهواز، مركز تحقيقات توانبخشى عضلانى اسكلتى.

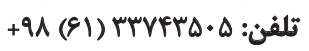
راياماهـ: baharlooh@gmail.com 


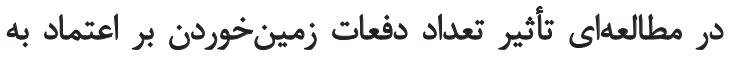

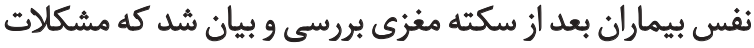

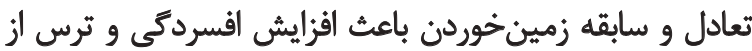

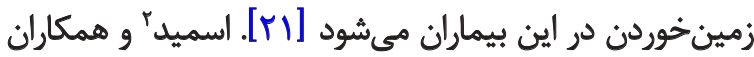

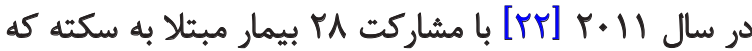

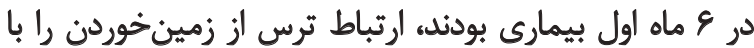

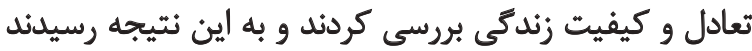

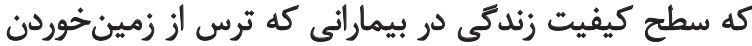

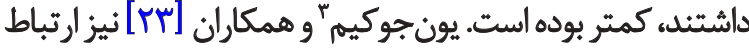

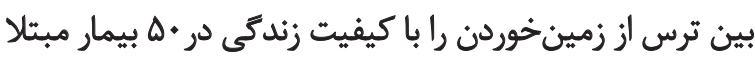

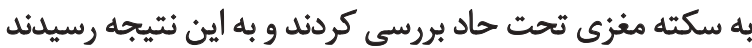

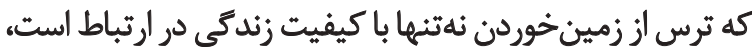

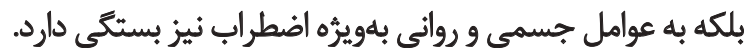
مطالعات محدودى درباره ترس از زمين خوردن هيس از سكته

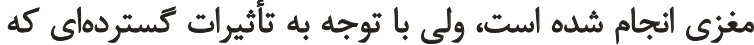

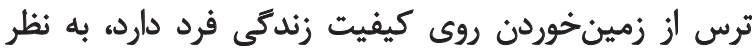

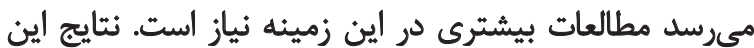

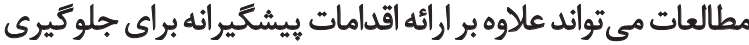

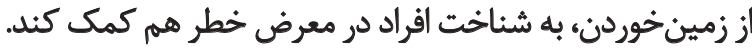

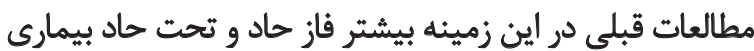

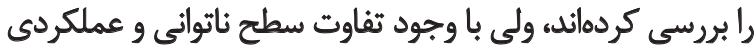

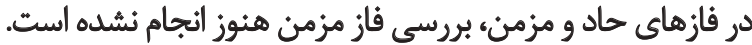

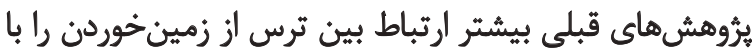

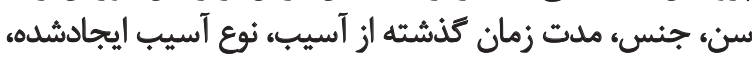

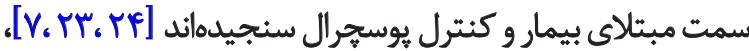

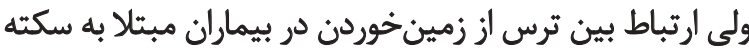

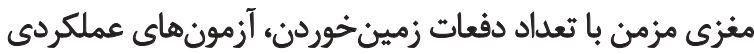

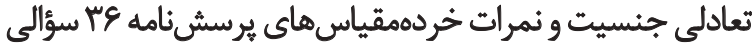

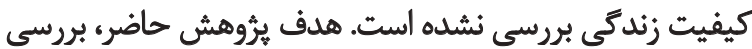

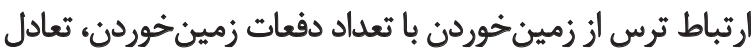
و كيفيت زندكى بيمار ان مبتلا به سكته مغزى مزمن است تعاد

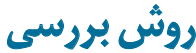

در اين مطالعه كه از نوع همبستگى بود، ff بيمار مبتلا به به بيه

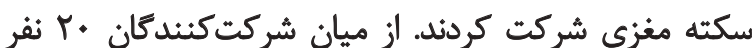

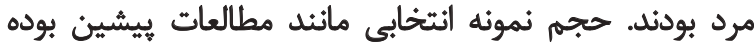

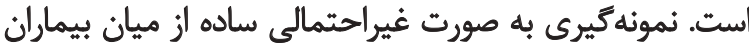

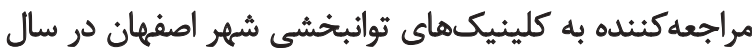

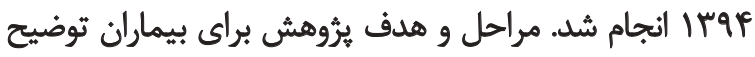

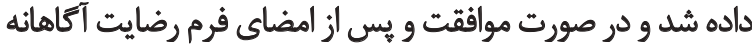
وارد يُروهش شدند. اين مطالعه با مجوز كميته اخلاق دانشكاه فران
مقلمه

سكته مغزى از مهمترين دلايل ناتوانى در بيشتر كشورها به به بهار

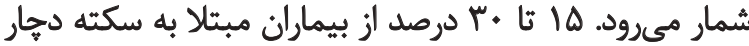

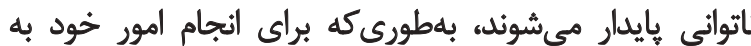

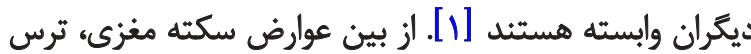

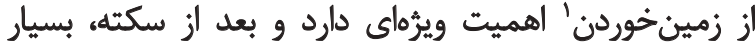

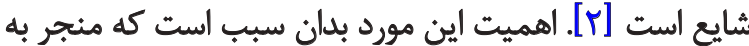

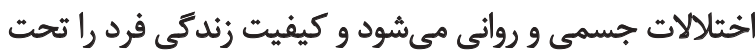

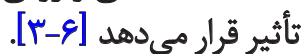

ترس از زمين خوردن مفهومى جدا از زمين خُوردن است و درد

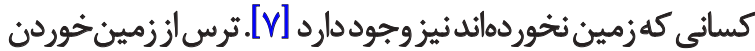

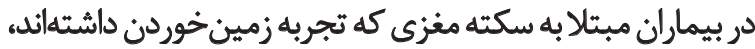

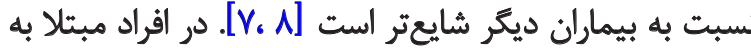

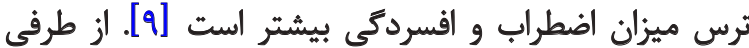

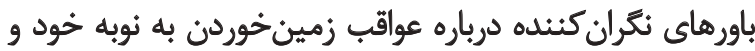

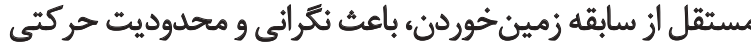

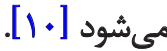

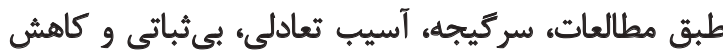

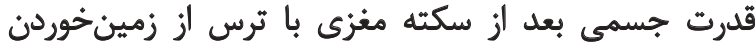

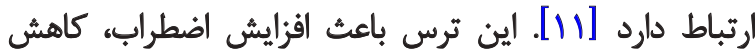

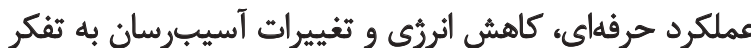

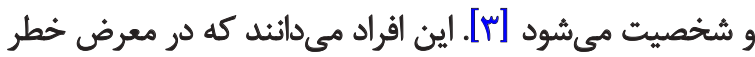

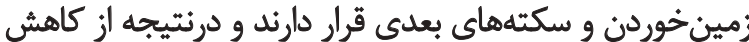

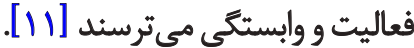

به نظر مهرسد ترس از زمينخوردن با تركيبى از عوامل

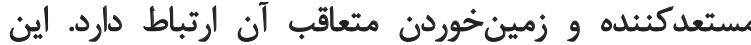

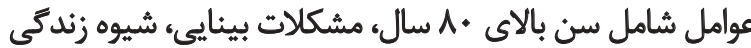

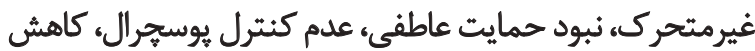

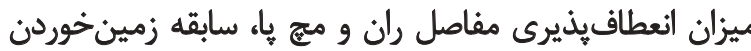

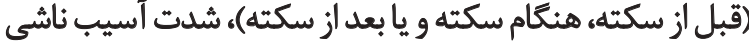

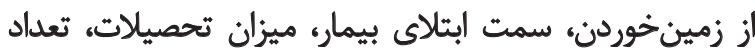

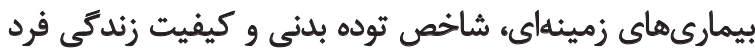

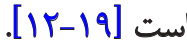

به صورت بالقوه، ترس از زمينخوردن نهتنها مي تواند كيفيت

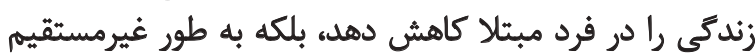

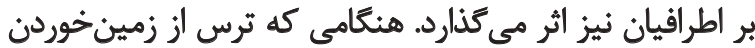

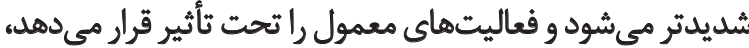

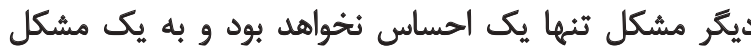

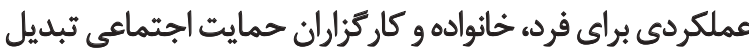

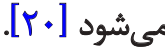


خط كش روى ديوار به موازات دست او ثابت مي شيود و عدد مقابل

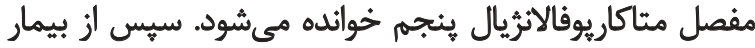

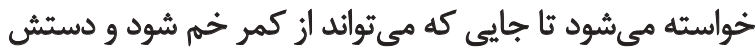

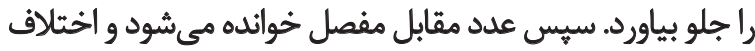

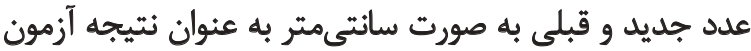

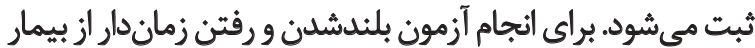

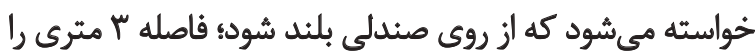

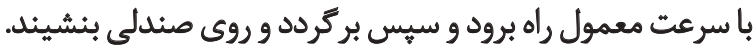

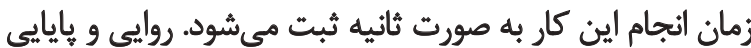

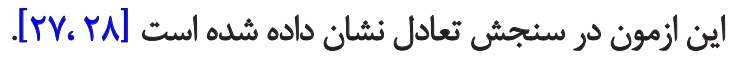

در اين مطالعه براى بررسى ارتباط ترس از زمين خوردن با بعداد

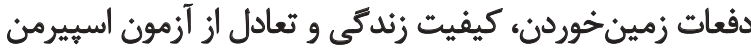

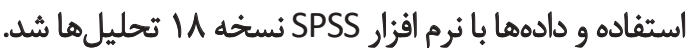

يافتهها

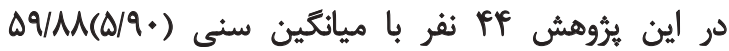

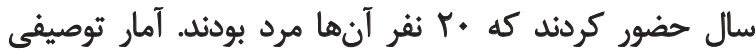

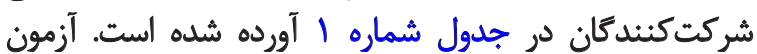

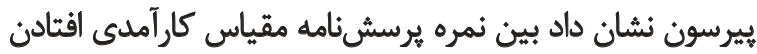

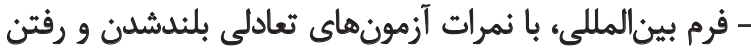

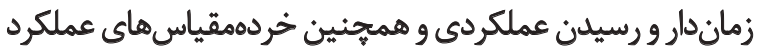

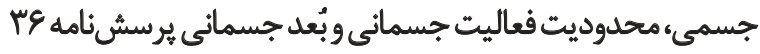

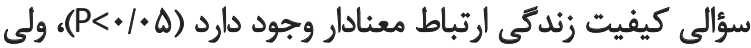

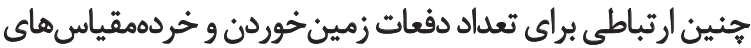

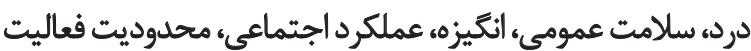

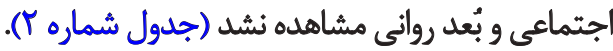

بحث

يافتههاى اين مطالعه نشان داد بين ترس از زمين خوردن بان

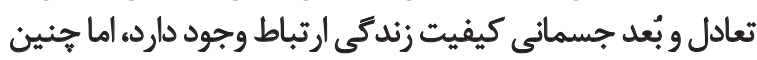

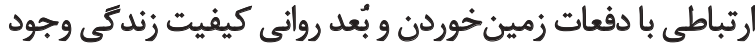

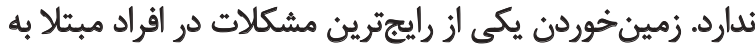

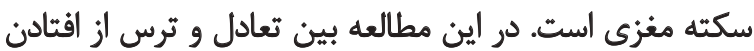

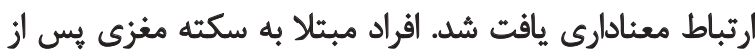

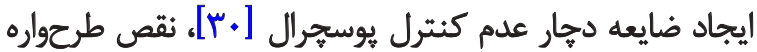

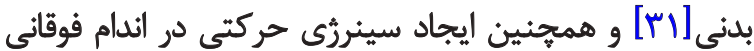

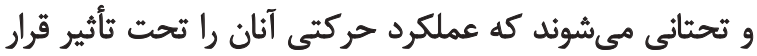

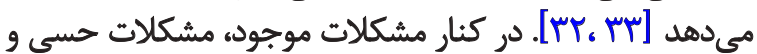

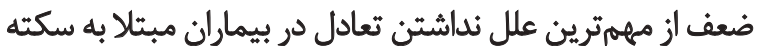

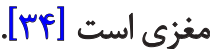

مطالعات كذشته نشان مىدهد مشكلات تعادلى با سابقه

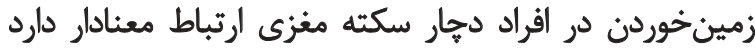

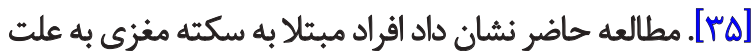

$$
\text { علوم يُرُشكى اصفهان انجام شده است. }
$$

معيارهاى ورود به مطالعه شامل كذشتن ع ماه از سكته مغزى،

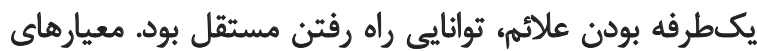

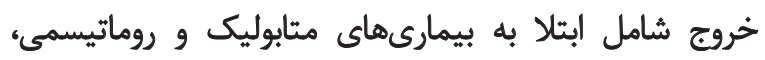

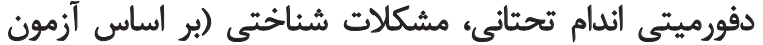

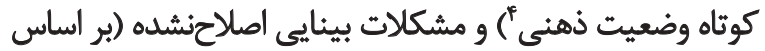

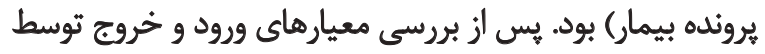

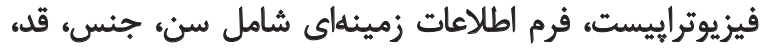

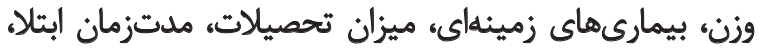

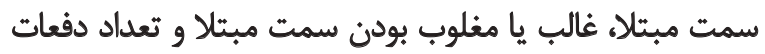
زمينخوردن در يك سال تذشته تكميل شد.

در اين يزوهش علاوه بر اطلاعات زمينهاي، ميزان ترس از

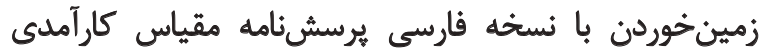

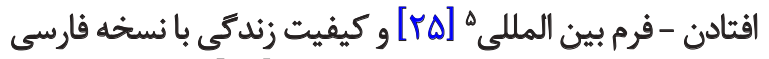

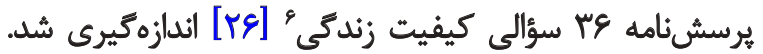

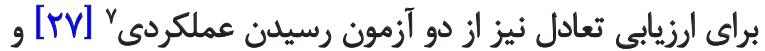

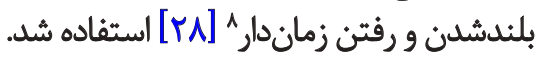

يرسش نامه مقياس كارآمدى افتادن - فرم بينالمللى شامل فيل فئل

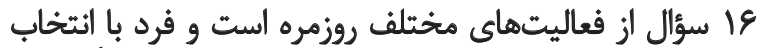

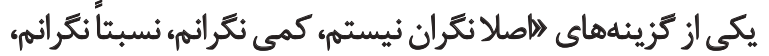

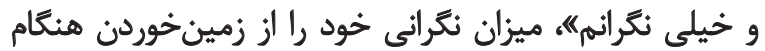

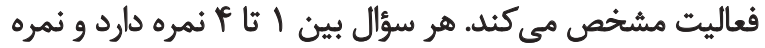

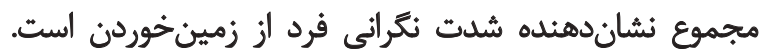

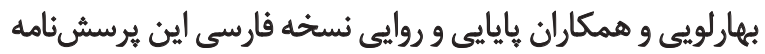

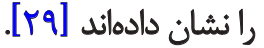

يرسشنامه عب سؤالى كيفيت زندگى براى ارزيابى كيفيت

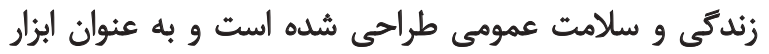

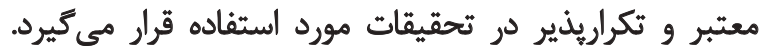

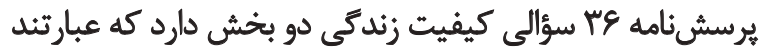

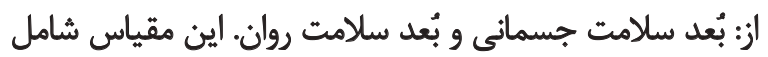

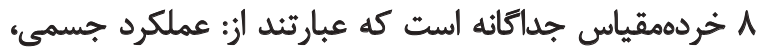

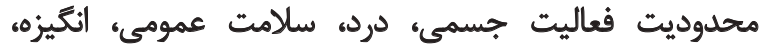

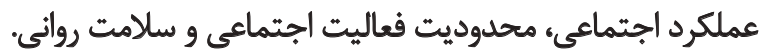

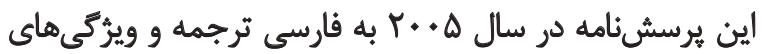

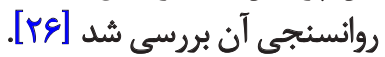
براى انجام آزمون رسيدن عملكردى، بيمار نزديك ديوار بازئ

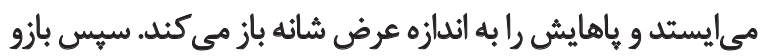

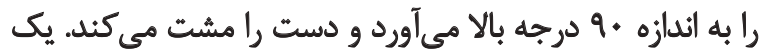

8. Timed Up and Go (TUG) 
جدول ا. آمار توصيفى شركت كنيدكّان

\begin{tabular}{|c|c|c|c|}
\hline انحراف معيار & ميائكين & متغير & \\
\hline$r / v$ & Te/ge & شاخص تودهه بلدنى & \\
\hline WAT & TV/AS & مدثت البئلا (ماه) & \\
\hline $1 / 89$ & $r / P V$ & تعلاد دفعات زمينخوردن & \\
\hline $1 . / A V$ & $\pi / \pi$ & بلندشدن و رقّن زماندار & \\
\hline$\Delta / \notin \varepsilon$ & WVE & آزمون رسيدن عملكردى & \\
\hline $1 . / 9 T$ & $r V / V$ & مقياس كارآمدى القتادن - فرم بين المللى & \\
\hline Tr/QS & $r a / .$. & عملكرد جسمى & \multirow{10}{*}{ رسش ثامه عب سؤالى كيفيت زندكى } \\
\hline $1 w / W$ & $r \Delta / r q$ & مهدوديث فعاليت جسمى & \\
\hline$r / q \Delta$ & DV/AT & درد & \\
\hline$r \cdot / r a$ & ex/ar & سلامت عمومى & \\
\hline$M / M$ & $\Delta Q / F$ & انكيزه & \\
\hline$r q / \pi T$ & QVAT & عملكرداجتماعى & \\
\hline 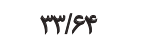 & $\Delta V / .$. & محلدوديت فعاليت الجتماعى & \\
\hline$r M / I Y$ & 91/90 & سلامث رواثيى & \\
\hline IV/A. & $\Delta . / M$ & لُعل جِسمى & \\
\hline Tr/qY & $\Delta Y / Q \varphi$ & لبُعل روانثى & \\
\hline
\end{tabular}

توانبخننى

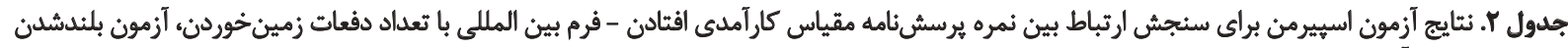

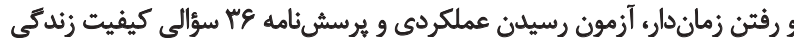

\begin{tabular}{|c|c|c|c|}
\hline ميزان معنادارى (P) & ميزان همبستكى (r) & & \\
\hline$+/ M$ & .1 .8 & تعلداد دفعات زمين خوردن & \\
\hline $1.1^{\circ}$ &.$/ P a$ & بلمندشن و رقتن زمانهار & \\
\hline.$/ .4$ &.$- / 4 *$ & آزهون رسيلن عملكردى & \\
\hline$<+\cdots 1^{*}$ &.$- / 4 a$ & عملكرد جسمى & \multirow{10}{*}{ برسش نامه عاب سؤالى كيفيت زندكى } \\
\hline$<++1^{\bullet}$ &.$- / \Delta$ & محلوديت فعاليت جسمى & \\
\hline . M & -.1 .8 & درد & \\
\hline.$/ M$ & $-\cdot / T I$ & مسلامت عمومى & \\
\hline .1 & $-+/ 7 q$ & انكيزه & \\
\hline.$/ \pi$ & $-+/ 18$ & 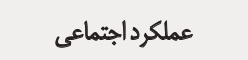 & \\
\hline.$/ 4$ & $-\cdot / 1 r$ & محدوديت فعاليت اجتماعي & \\
\hline t/M & -+1.9 & سلامت روائى & \\
\hline$<+\cdots$ & $-r / \Delta T$ & بعل جسمى & \\
\hline.$/ \% q$ & $-+/ 10$ & بعل رواثى & \\
\hline
\end{tabular}


بر خلاف مطالعه موجوده در برخى مطالعات ارتباطى بين

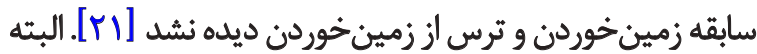

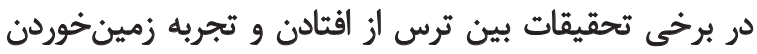

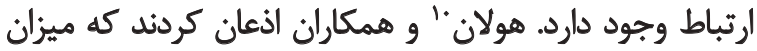

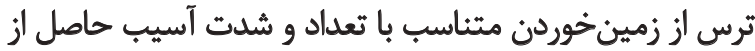

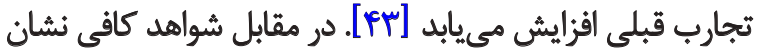

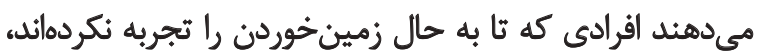

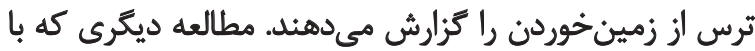

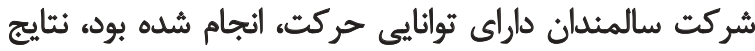

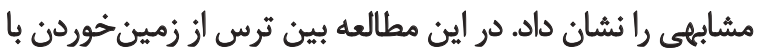

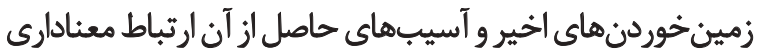

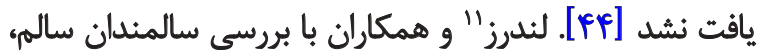

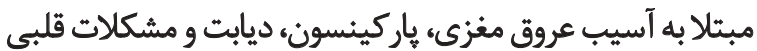

عروقى به نتايج مشابه رسيدند [FD] ئاركين.

تثيجه كَيرى

يافتههاى اين يُروهش نشان مى دهد در افراد مبتلا به سكته

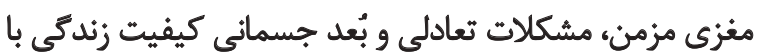

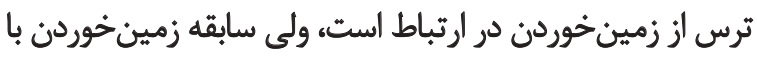
ترس از زمين ازخوردن ارتباطى ندارد.

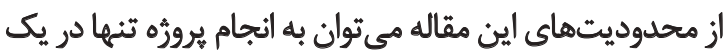

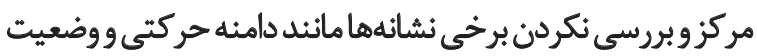

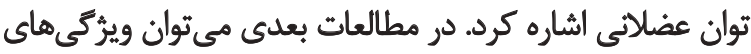

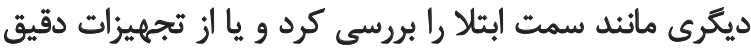

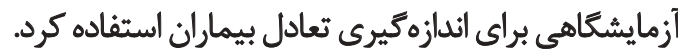

$$
\text { تشيكر و قبدرداني }
$$

اين مقاله حاصل طرحى با حمايت مركز تحقيقات اختلالات اسكلتى عضلانى دانشكاه علوم يزشكى اصفيت اصفهان است

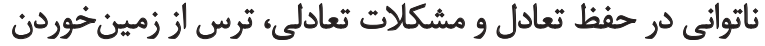

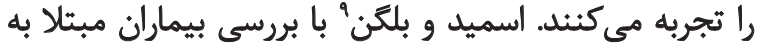

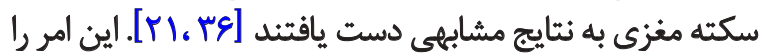

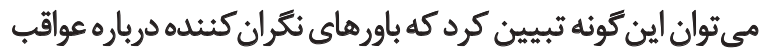

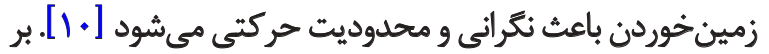

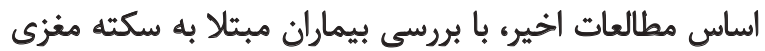

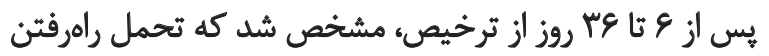

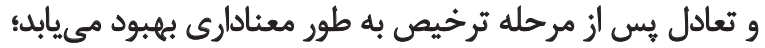

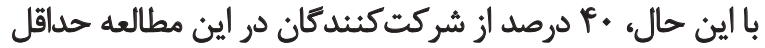

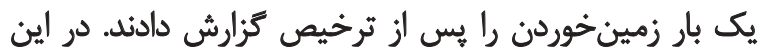

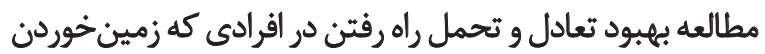

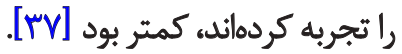

مطالعه ديكرى نيز نشان داد ترس از زمين خوردن علاوه بر سن،

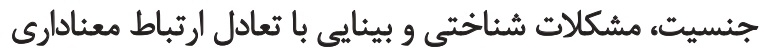

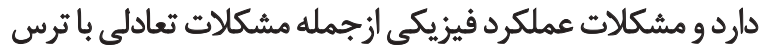

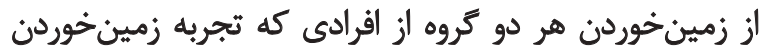

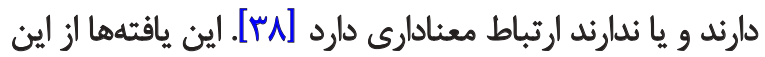

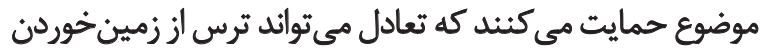

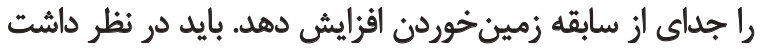

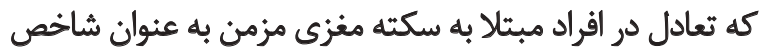

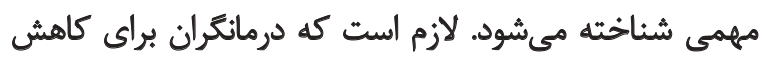

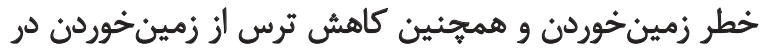

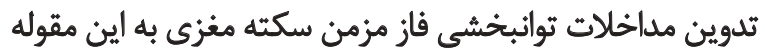
توجه ويثٔاي داشته باشند.

نتايج اين مطالعه نشان داد ثرس از زمين خوردن با كاهش

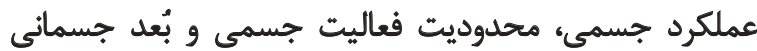

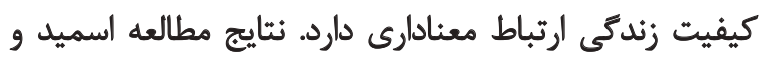

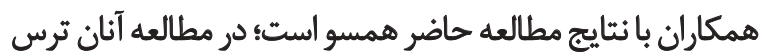

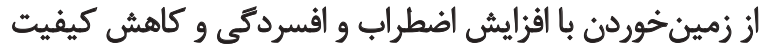

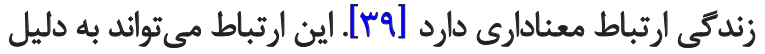

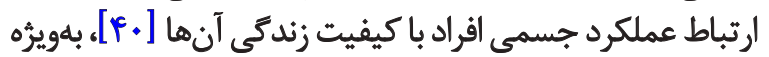

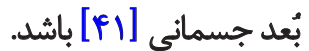

يك مطالعه آيندهنكر نشان مى دهد كه ترس از زمين خوردن

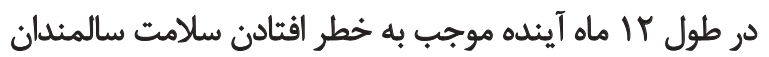

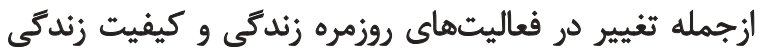

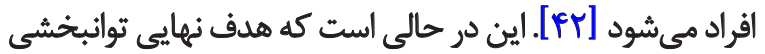

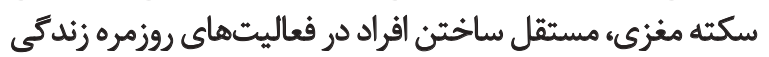

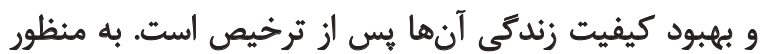

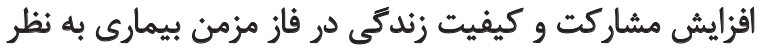

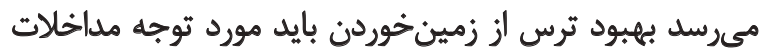

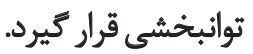




\section{References}

[1] Goldstein LB, Adams R, Alberts MJ, Appel LJ, Brass LM, Bushnell CD, et al. Primary prevention of ischemic stroke. Stroke. 2006; 37(6):1583-633. doi: 10.1161/01.str.0000223048.70103.f1

[2] Jorgensen L, Engstad T, Jacobsen BK. Higher incidence of falls in long-term stroke survivors than in population controls: depressive symptoms predict falls after stroke. Stroke. 2002; 33(2):5427. doi: $10.1161 / \mathrm{hs} 0202.102375$

[3] Schmid AA, Acuff M, Doster K, Gwaltney-Duiser A, Whitaker A, Damush T, et al. Poststroke fear of falling in the hospital setting. Topics in Stroke Rehabilitation. 2009; 16(5):357-66. doi: $10.1310 / \operatorname{tsr} 1605-357$

[4] Scheffer AC, Schuurmans MJ, van Dijk N, van der Hooft T, de Rooij SE. Fear of falling: Measurement strategy, prevalence, risk factors and consequences among older persons. Age and Ageing. 2008; 37(1):19-24. doi: 10.1093/ageing/afm169

[5] Tinetti ME, Powell L. 4 Fear of falling and low self-efficacy: A cause of dependence in elderly persons. Journal of Gerontology. 1993; 48(Special):35-8. doi: 10.1093/geronj/48.special_issue.35

[6] Vellas B, Cayla F, Bocquet H, De Pemille F, Albarede JL. Prospective study of restriction of acitivty in old people after falls. Age and Ageing. 1987; 16(3):189-93. doi: 10.1093/ageing/16.3.189

[7] Legters K. Fear of falling. Physical Therapy. 2002; 82(3):264-72. PMID: 11869155

[8] Howland J, Lachman ME, Peterson EW, Cote J, Kasten L, Jette A. Covariates of fear of falling and associated activity curtailment. The Gerontologist. 1998; 38(5):549-55. doi: 10.1093/geront/38.5.549

[9] Chou KL, Yeung FKC, Wong ECH. Fear of falling and depressive symptoms in Chinese elderly living in nursing homes: Fall efficacy and activity level as mediator or moderator. Aging \& Mental Health. 2005; 9(3):255-61. doi: 10.1080/13607860500114035

[10] Delbaere K, Crombez G, van Haastregt JCM, Vlaeyen JWS. Falls and catastrophic thoughts about falls predict mobility restriction in community-dwelling older people: A structural equation modelling approach. Aging \& Mental Health. 2009; 13(4):587-92. doi: $10.1080 / 13607860902774444$

[11] SchmidAA, Rittman M. Fear of falling: An emerging issue after stroke. Topics in Stroke Rehabilitation.2007; 14(5):46-55. doi: $10.1310 /$ tsr1405-46

[12] Tinetti ME, Speechley M, Ginter SF. Risk factors for falls among elderly persons living in the community. New England Journal of Medicine. 1988; 319(26):1701-7. doi: 10.1056/ neim198812293192604

[13] Vellas BJ, Wayne SJ, Romero LJ, Baumgartner RN, Garry PJ. Fear of falling and restriction of mobility in elderly fallers. Age and Ageing. 1997; 26(3):189-93. doi: 10.1093/ageing/26.3.189

[14] Friedman SM, Munoz B, West SK, Rubin GS, Fried LP. Falls and fear of falling: which comes first? a longitudinal prediction model suggests strategies for primary and secondary prevention. Journal of the American Geriatrics Society. 2002; 50(8):1329-35. doi: 10.1046/j.1532-5415.2002.50352.x
[15] Murphy SL, Williams CS, Gill TM. Characteristics associated with fear of falling and activity restriction in community-living older persons. Journal of the American Geriatrics Society. 2002; 50(3):516-20. doi: 10.1046/j.1532-5415.2002.50119.x

[16] Murphy SL, Dubin JA, Gill TM. The development of fear of falling among community-living older women: predisposing factors and subsequent fall events. The Journals of Gerontology Series A: Biological Sciences and Medical Sciences. 2003; 58(10):M943-7. doi: 10.1093/gerona/58.10.m943

[17] Kressig RW, Wolf SL, Sattin RW, O’Grady M, Greenspan A, Curns A, et al. Associations ofdemographic, functional, and behavioral characteristics with activity-related fear of falling among older adults transitioning to frailty. Journal of the American Geriatrics Society. 2001; 49(11):1456-62. doi: 10.1046/j.15325415.2001.4911237.x

[18] Arfken CL, Lach HW, Birge SJ, Miller JP. The prevalence and correlates of fear of falling in elderly persons living in the community. American Journal of Public Health. 1994; 84(4):565-70. doi: $10.2105 /$ ajph. 84.4 .565

[19] Lachman ME, Howland J, Tennstedt S, Jette A, Assmann S, Peterson EW. Fear of falling and activity restriction: The Survey of Activities and Fear of falling in the Elderly (SAFE). The Journals of Gerontology Series B: Psychological Sciences and Social Sciences. 1998; 53B(1):P43-50. doi: 10.1093/geronb/53b.1.p43

[20] Perez Jara J, Walker D, Heslop P, Robinson S. Measuring fear of falling and its effect on quality of life and activity. Reviews in Clinical Gerontology. 2010; 20(04):277-87. doi: 10.1017/ s0959259810000237

[21] Belgen B, Beninato M, Sullivan PE, Narielwalla K. The association of balance capacity and falls self-efficacy with history of falling in community-dwelling people with chronic stroke. Archives of Physical Medicine and Rehabilitation. 2006; 87(4):554-61. doi 10.1016/j.apmr.2005.12.027

[22] Schmid AA, Van Puymbroeck M, Knies K, Spangler-Morris C, Watts K, Damush T, et al. Fear of falling among people who have sustained a stroke: a 6-month longitudinal pilot study. American Journal of Occupational Therapy. 2011; 65(2):125-32. doi: 10.5014/ajot.2011.000737

[23] Kim EJ, Kim DY, Kim WH, Lee KL, Yoon YH, Park JM, et al. Fear of falling in subacute hemiplegic stroke patients: associating factors and correlations with quality of life. Annals of Rehabilitation Medicine. 2012; 36(6):797. doi: 10.5535/arm.2012.36.6.797

[24] Simpson LA, Miller WC, Eng JJ. Effect of stroke on fall rate, location and predictors: a prospective comparison of older adults with and without stroke. PLoS ONE. 2011; 6(4):e19431. doi: 10.1371/journal.pone.0019431

[25] Baharlouei H, Salavati M, Akhbari B, Mosallanezhad Z, Mazaheri M, Negahban H. Cross-cultural validation of the Falls Efficacy Scale International (FES-I) using self-report and interviewbased questionnaires among Persian-speaking elderly adults. Archives of Gerontology and Geriatrics. 2013; 57(3):339-44. doi: 10.1016/j.archger.2013.06.005

[26] Montazeri A, Goshtasebi A, Vahdaninia M, Gandek B. The Short Form health survey (SF-36): Translation and validation study of the Iranian version. Quality of Life Research. 2005; 14(3):875-82. doi: 10.1007/s11136-004-1014-5 
[27] Duncan PW, Weiner DK, Chandler J, Studenski S. Functional reach: A new clinical measure of balance. Journal of Gerontology. 1990; 45(6):M192-M197. doi: 10.1093/geronj/45.6.m192

[28] Shumway Cook A, Brauer S, Woollacott M. Predicting the probability for falls in community-dwelling older adults using the Timed Up \& Go Test. Physical Therapy. 2000; 80(9):896-903. PMID: 10960937

[29] Baharlouei H, Salavati M, Akhbari B, Mosallanezhad Z, Mazaheri M, Negahban H. Cross-cultural validation of the Falls Efficacy Scale International (FES-I) using self-report and interviewbased questionnaires among Persian-speaking elderly adults. Archives of Gerontology and Geriatrics. 2013; 57(3):339-44. doi: 10.1016/j.archger.2013.06.005

[30] Perlmutter S, Lin F, Makhsous M. Quantitative analysis of static sitting posture in chronic stroke. Gait \& Posture. 2010; 32(1):53 6. doi: $10.1016 /$ j.gaitpost.2010.03.005

[31] Corbett A, Shah S. Body scheme disorders following stroke and assessment in occupational therapy. British Journal of Occupational Therapy. 1996; 59(7):325-9. doi: 10.1177/030802269605900709

[32] Dipietro L, Krebs HI, Fasoli SE, Volpe BT, Stein J, Bever C, et al. Changing motor synergies in chronic stroke. Journal of Neurophysiology. 2007; 98(2):757-68. doi: 10.1152/jn.01295.2006

[33] Abdollahi I, Joghataei MT, Salavati M, Ebrahimi E, A'shayeri H. Explicit learning of a perceptual-motor skill after stroke by using affected hand. Journal of Rehabilitation. 2007; 8(2):24-30.

[34] Tyson SF, Hanley M, Chillala J, Selley A, Tallis RC. Balance disability after stroke. Physical Therapy. 2006; 86(1):30-8. doi: $10.1093 / \mathrm{ptj} / 86.1 .30$

[35] Mackintosh SF, Hill KD, Dodd KJ, Goldie PA, Culham EG. Balance score and a history of falls in hospital predict recurrent falls in the 6 months following stroke rehabilitation. Archives of Physical Medicine and Rehabilitation. 2006; 87(12):1583-9. doi: 10.1016/j.apmr.2006.09.004

[36] Schmid AA, Arnold SE, Jones VA, Jane Ritter M, Sapp SA, Van Puymbroeck M. Fear of falling in people with chronic stroke. American Journal of Occupational Therapy. 2015; 69(3):6903350020p1. doi: 10.5014/ajot.2015.016253

[37] Blennerhassett JM, Dite W, Ramage ER, Richmond ME. Changes in balance and walking from stroke rehabilitation to the community: a follow-up observational study. Archives of Physical Medicine and Rehabilitation. 2012; 93(10):1782-7. doi: 10.1016/j. apmr.2012.04.005

[38] Andersson ÅG, Kamwendo K, Appelros P. Fear of falling in stroke patients: Relationship with previous falls and functional characteristics. International Journal of Rehabilitation Research. 2008; 31(3):261-4. doi: 10.1097/mrr.0b013e3282fba390

[39] Schmid AA, Van Puymbroeck M, Knies K, Spangler Morris C, Watts K, Damush T, et al. Fear of falling among people who have sustained a stroke: a 6-month longitudinal pilot study. American Journal of Occupational Therapy. 2011; 65(2):125-32. doi: 10.5014/ajot.2011.000737

[40] El Khoury F, Cassou B, Latouche A, Aegerter P, Charles M-A, Dargent Molina P. Effectiveness of two year balance training programme on prevention of fall induced injuries in at risk women aged 75-85 living in community: Ossébo randomised controlled trial. BMJ. 2015; h3830. doi: 10.1136/bmj.h3830

[41] Trombetti A, Reid KF, Hars M, Herrmann FR, Pasha E, Phillips EM, et al. Age-associated declines in muscle mass, strength, power, and physical performance: Impact on fear of falling and quality of life. Osteoporosis International. 2015; 27(2):463-71. doi: 10.1007/s00198-015-3236-5

[42] Cumming RG, Salkeld G, Thomas M, Szonyi G. Prospective study of the impact of fear of falling on activities of daily living, sf-36 scores, and nursing home admission. The Journals of Gerontology Series A: Biological Sciences and Medical Sciences. 2000; 55(5):M299-M305. doi: 10.1093/gerona/55.5.m299

[43] Howland J, Peterson EW, Levin WC, Fried L, Pordon D, Bak S. Fear of falling among the community-dwelling elderly. Journal of Aging and Healt. 1993; 5(2):229-43. doi: $10.1177 / 089826439300500205$

[44] Myers AM, Powell LE, Maki BE, Holliday PJ, Brawley LR, Sherk W. Psychological indicators of balance confidence: Relationship to actual and perceived abilities. The Journals of Gerontology Series A: Biological Sciences and Medical Sciences. 1996; 51A(1):M37-43. doi: 10.1093/gerona/51a.1.m37

[45] Landers MR, Oscar S, Sasaoka J, Vaughn K. Balance confidence and fear of falling avoidance behavior are most predictive of falling in older adults: prospective analysis. Physical Therapy. 2015; 96(4):433-42. doi: 10.2522/ptj.20150184 